\title{
Effect of intraperitoneal local anesthesia on enhanced recovery outcomes after bariatric surgery: a randomized controlled pilot study
}

\author{
Amer Jarrar, MD \\ Naveen Eipe, MD \\ Robert Wu, MD \\ Amy Neville, MD, MSc \\ Jean-Denis Yelle, MD \\ Joseph Mamazza, MD
}

Accepted November 30, 2020

\author{
Correspondence to: \\ A. Jarrar \\ Department of Surgery \\ Faculty of Medicine \\ University of Ottawa \\ CPC R331, 1053 Carling Ave \\ Ottawa ON K1H $8 \mathrm{M} 5$ \\ ajarrar.md@gmail.com
}

Cite as: Can J Surg 2021 November 10; 64(6). doi: 10.1503/cjs.017719

\begin{abstract}
Background: Patients with extreme obesity are at high risk for adverse perioperative events, especially when opioid-centric analgesic protocols are used, and perioperative pain management interventions in bariatric surgery could improve safety, outcomes and satisfaction. We aimed to evaluate the impact of intraperitoneal local anesthesia (IPLA) on enhanced recovery after bariatric surgery (ERABS) outcomes.
\end{abstract}

Methods: We conducted a prospective double-blind randomized controlled pilot study in adherence to an a priori peer-reviewed protocol. Patients undergoing laparoscopic Roux-en-Y gastric bypass surgery (LRYGB) with an established ERABS protocol between July 2014 and February 2015 were randomly allocated to receive either IPLA with $0.2 \%$ ropivacaine (intervention group) or normal saline (control group). We measured pain scores, analgesic consumption and adverse effects. Functional prehabilitation outcomes, including peak expiratory flow (PEF) and the Six Minute Walk Test (6MWT) and Quality of Recovery Survey-40 (QoR-40) scores, were assessed before surgery, and 1 day and 7 days postoperatively.

Results: One hundred patients were randomly allocated to the study groups, of whom 92 completed the study, 46 in each group. There were no statistically significant differences between the 2 groups in baseline characteristics or any primary or secondary outcomes. Pain scores and analgesic consumption were low in both groups. There were no adverse events. Significant declines in PEF and 6MWT and QoR-40 scores were noted on postoperative day 1 in both groups; the values returned to baseline on postoperative day 7 in both groups.

Conclusion: Intraperitoneal local anesthesia with ropivacaine did not reduce postoperative pain or analgesic consumption when administered intraoperatively to patients undergoing LRYGB. Standardization of the ERABS protocol benefited patients, with functional prehabilitation outcomes returning to baseline postoperatively. Trial registration: ClinicalTrials.gov no. NCT 02154763

Contexte : Les patients qui souffrent d'obésité extrême sont exposés à un risque élevé de complications périopératoires, surtout quand on a recours à des protocoles antalgiques axés sur les opioïdes, et certaines interventions périopératoires de gestion de la douleur pour les chirurgies bariatriques pourraient améliorer la sécurité, les résultats et la satisfaction. Nous avons voulu évaluer l'impact de l'anesthésie locale intrapéritonéale (ALIP) sur l'amélioration des paramètres du rétablissement (ou ERABS, pour enhanced recovery after bariatric surgery) post-chirurgie bariatrique.

Méthodes : Nous avons procédé à une étude pilote prospective à double insu randomisée et contrôlée sur un protocole préalablement révisé par des pairs. Les patients soumis à une intervention pour dérivation gastrique Roux-en-Y par laparoscopie avec un protocole ERABS, entre juillet 2014 et février 2015 ont été assignés aléatoirement soit à l'ALIP avec ropivacaïne à $0,2 \%$ (groupe sous intervention), soit à une solution physiologique normale (groupe témoin). Nous avons mesuré les scores de douleur, la prise d'analgésiques et les effets indésirables. Les paramètres fonctionnels préadaptation, incluant le débit expiratoire de pointe (DEP) et les scores au test de marche de 6 minutes (6MWT) et au questionnaire d'évaluation de la qualité du rétablissement en 40 points (QoR-40) ont été évalués aux jours postopératoires 1 et 7.

Résultats : Cent patients ont été assignés aléatoirement aux groupes d'étude, dont 92 ont mené l'étude à terme, 46 dans chaque groupe. On n'a noté aucune différence statistiquement significative entre les 2 groupes pour ce qui est des caractéristiques de départ et des paramètres primaires ou secondaires. Les scores de douleur et la prise d'analgésiques étaient bas dans les 2 groupes. On n'a observé aucun effet indésirable. 


\begin{abstract}
On a noté des baisses significatives du DEP et des scores au test 6MWT et au questionnaire QoR-40 au jour postopératoire 1 dans les 2 groupes; au jour postopératoire 7 , dans les 2 groupes, les valeurs étaient revenues à celles qu'elles étaient au départ.
\end{abstract}

Conclusion : L'anesthésie locale intrapéritonéale avec la ropivacaïne n'a réduit ni la douleur ni la prise d'analgésiques après la chirurgie lorsqu'elle était administré durant l'intervention à des patients soumis à une dérivation gastrique Roux-en-Y par laparoscopie. La standardisation du protocole ERABS a été bénéfique aux patients, et après l'opération, les paramètres de préadaptation fonctionnelle revenaient aux valeurs de départ. Numéro d'enregistrement de la recherche : ClinicalTrials.gov, no. NCT 02154763

I mplementation of Enhanced Recovery After Surgery (ERAS) in a wide variety of surgical models is well established and has shown the importance of multimodal strategies in improving perioperative patient safety and outcomes. ${ }^{1}$ Patients undergoing elective weight loss (bariatric) surgery are at risk for increased complications owing to their obesity-related comorbidity. Enhanced recovery after bariatric surgery (ERABS) may be critical in this context for a variety of clinical reasons. ${ }^{2}$ For example, if ERABS interventions can improve the patient's breathing and coughing ability, and ensure early oral intake or adequate ambulation, these outcomes could substantially reduce the risk of the major causes of morbidity and mortality related to bariatric surgery, including cardiorespiratory complications, deep vein thrombosis and pulmonary embolism. ${ }^{3}$

Pain management remains particularly challenging in patients with extreme obesity. When coexisting cardiorespiratory illnesses and the high prevalence of obstructive sleep apnea are combined with poorly or inappropriately managed pain, further perioperative complications can occur in this patient population. ${ }^{3}$ Traditional opioid-centric pain management protocols increase sedation, respiratory depression, nausea and vomiting, all of which can adversely affect recovery, early discharge and return to baseline function. ${ }^{3}$ Effective perioperative pain management is one of the cornerstones of ERABS. Early recovery after bariatric surgery provides the framework to improve patient safety and outcomes with protocol standardization and multimodal interventions. ${ }^{2,4}$ Implementing multimodal nonopioid analgesia protocols and exploring regional anesthesia techniques are therefore important to achieving ERABS outcomes. These advances in perioperative care can potentially be applied to the wider population of patients with extreme obesity undergoing a variety of surgical procedures. ${ }^{4}$

Intraperitoneal local anesthesia (IPLA) has been studied extensively in nonbariatric general surgery and gynecology. ${ }^{5,6}$ In bariatric surgery, IPLA with bupivacaine has been reported to reduce pain and analgesic consumption. ${ }^{7,8}$ We conducted a prospective double-blind randomized controlled pilot study to evaluate the clinical efficacy of adding IPLA using ropivacaine to a standardized ERABS protocol ${ }^{9}$ to improve pain management and functional outcomes in patients undergoing elective bariatric surgery. We chose to study ropivacaine because it may be clinically more efficacious and have a better safety profile in locoregional anesthesia than bupivacaine. ${ }^{10,11}$

\section{Methods}

The research protocol was approved by the Ottawa Health Science Network Research Ethics Board (OHREB 20120743-01H) and registered with ClinicalTrials.gov (NCT 02154763) as a randomized double-blind placebocontrolled trial. The protocol for the trial was then peer reviewed and published a priori. ${ }^{9}$ Approval for the use of the study drug (ropivacaine) as an IPLA was deemed offlabel. We obtained federal health care authority approval for the study (Health Canada no. 160184) before patient enrolment.

\section{Participants}

Patients undergoing laparoscopic Roux-en-Y gastric bypass for obesity at the regional Bariatric Centre of Excellence (The Ottawa Hospital) between July 2014 and February 2015 were approached by a participating surgeon or a nurse from The Ottawa Hospital Bariatric Surgery Clinic for participation. If the patient agreed to participate, consent was obtained by a member of the research team (A.J.) who was independent from the clinical care of patients.

We included all adults (age $>18 \mathrm{yr}$ ) who were able to tolerate general anesthesia and pneumoperitoneum, and to provide informed consent for the surgery. Patients with chronic pain requiring preoperative opioids were included. Exclusion criteria were planned sleeve gastrectomy (intraoperative conversion to sleeve gastrectomy after IPLA delivery was included and analyzed with the intent-to-treat approach); allergy to local anesthetics; severe underlying cardiovascular disease, including congestive heart failure, conduction abnormalities and ischemic heart disease; chronic renal disease stage 3 or higher (defined as creatinine clearance $<60 \mathrm{~mL} / \mathrm{h}$ ); hepatic dysfunction Child-Pugh class B or $\mathrm{C}$; and previous foregut surgery, including esophageal, gastric, liver or pancreas resection. Inclusion and exclusion criteria are described in detail in the published protocol. ${ }^{9}$

The sample size for pilot trials is typically determined pragmatically. We aimed to randomly allocate 100 patients to the study groups. 


\section{Randomization}

Patients were assigned in a 1:1 ratio to either the intervention (IPLA) group or the control group by means of a simple computer-generated in-hospital randomization platform. The unblinded data were kept securely by pharmacy and were generated only a day before the operating day for each patient. The study was adequately and appropriately blinded: patients and providers (surgeons, anesthesiologists, operating room staff, nurses on both preand postsurgery units) involved in data collection and analysis had no knowledge of the group to which the patient was allocated.

The pharmacy independently prepared the treatment solution (ropivacaine or normal saline) in a standardized $100 \mathrm{~mL}$ bag. The treatment solution was attached to the patient's unique identifier and did not indicate to which study arm the patient was allocated. The intravenous bags were labelled according to Health Canada regulations and did not disclose the bag content. The treatment medication was delivered to the operating room on the day of surgery.

\section{Intervention}

All procedures were performed by 1 of 3 participating expert surgeons (J.M., J.-D.Y. or A.N.). All patients received acetaminophen ( $975 \mathrm{mg}$ orally) and celecoxib (400 mg orally) 2 hours before surgery. With standard monitoring, anesthesia was induced with propofol and fentanyl, administered intravenously and a neuromuscular blocking agent was administered to facilitate orotracheal intubation and controlled ventilation. After induction of anesthesia, all patients received ketamine $(20 \mathrm{mg})$ and dual antiemetic therapy (dexamethasone $8 \mathrm{mg}$, and ondansetron $8 \mathrm{mg}$ ) intravenously. Anesthesia was maintained with volatile agents via controlled ventilation using an air-oxygen mixture. A dexmedetomidine infusion $(0.4-0.7 \mu \mathrm{g} / \mathrm{kg} / \mathrm{h})$ was continued throughout anesthesia, and additional boluses of fentanyl were administered as required. No other anesthetic or analgesic infusions were administered intraoperatively.

A standardized surgical technique was used for this study. After the pneumoperitoneum was created and all trocars were placed, the standard suction/irrigation device was used to instill $100 \mathrm{~mL}$ of $0.2 \%$ ropivacaine or normal saline into the peritoneal cavity at the start of the case and before surgical dissection. Under direct visualization, $50 \mathrm{~mL}$ of ropivacaine or normal saline was infused over the esophageal hiatus, and the remaining $50 \mathrm{~mL}$ was infused throughout the abdomen. The remainder of the surgery and anesthesia followed standardized procedures and protocols. For postoperative pain control, patients were offered, on demand, acetaminophen, followed by ketorolac, then tramadol, and finally hydromorphone.

\section{Outcomes}

Participants provided their baseline demographic information, existing comorbidities, past medical and surgical history, medications, allergies and social history. Baseline function and peak expiratory flow (PEF) were measured, and the Six Minute Walk Test (6MWT) and Quality of Recovery Survey-40 (QoR-40) ${ }^{12}$ were administered. The primary outcome was postoperative pain as measured on a numeric pain scale ranging from 0 (no pain at all) to 10 (worst pain imaginable). We considered a 30\% reduction in pain in the intervention group compared to the control group a clinically significant improvement. The secondary outcomes included opioid analgesic use during the hospital stay, PEF, 6MWT result, impact on condition-specific quality of life (assessed with the QoR-40) and perioperative complications.

\section{Statistical analysis}

Data were collected as described in the study $\operatorname{protocol}^{9}$ and entered into a secure monitored database. We performed a multivariate analysis with the model adjusted for covariates such as age, gender, body mass index, pain syndromes and history of musculoskeletal diseases; we considered alcohol consumption, medication use and intraoperative concurrent procedures as time-fixed covariates. We described quantitative variables by mean and standard deviation (SD). Analgesic use was recorded in the postanesthesia care unit and at fixed time intervals until discharge. We analyzed repeated measures using a mixed modelling approach and performed an intention-to-treat analysis comparing the intervention and control groups for pain score, PEF, $6 \mathrm{MWT}$ score and QoR-40 score over time using SPSS version 18 for Windows (IBM Corp.).

\section{Results}

A total of 148 patients were approached, of whom 100 were randomly allocated to the study groups. Ninetytwo patients completed the study, 46 in each group (Figure 1). There were 77 women (84\%) and 15 men $(16 \%)$, with a mean body mass index of 47.2 (SD 6.4) and a mean age of 44.8 (SD 9.3) years. The preoperative characteristics and intraoperative variables for the 2 groups are presented in Table 1 . There was no statistically significant difference between the 2 groups except for mean body mass index and history of asthma, which were higher in the intervention group than in the control group. The prevalence of chronic pain was higher in the control group.

There was no statistically significant difference between the intervention group and the control group in pain scores at any postoperative time point (Table 2). The average pain scores remained consistently low for the entire study period. 


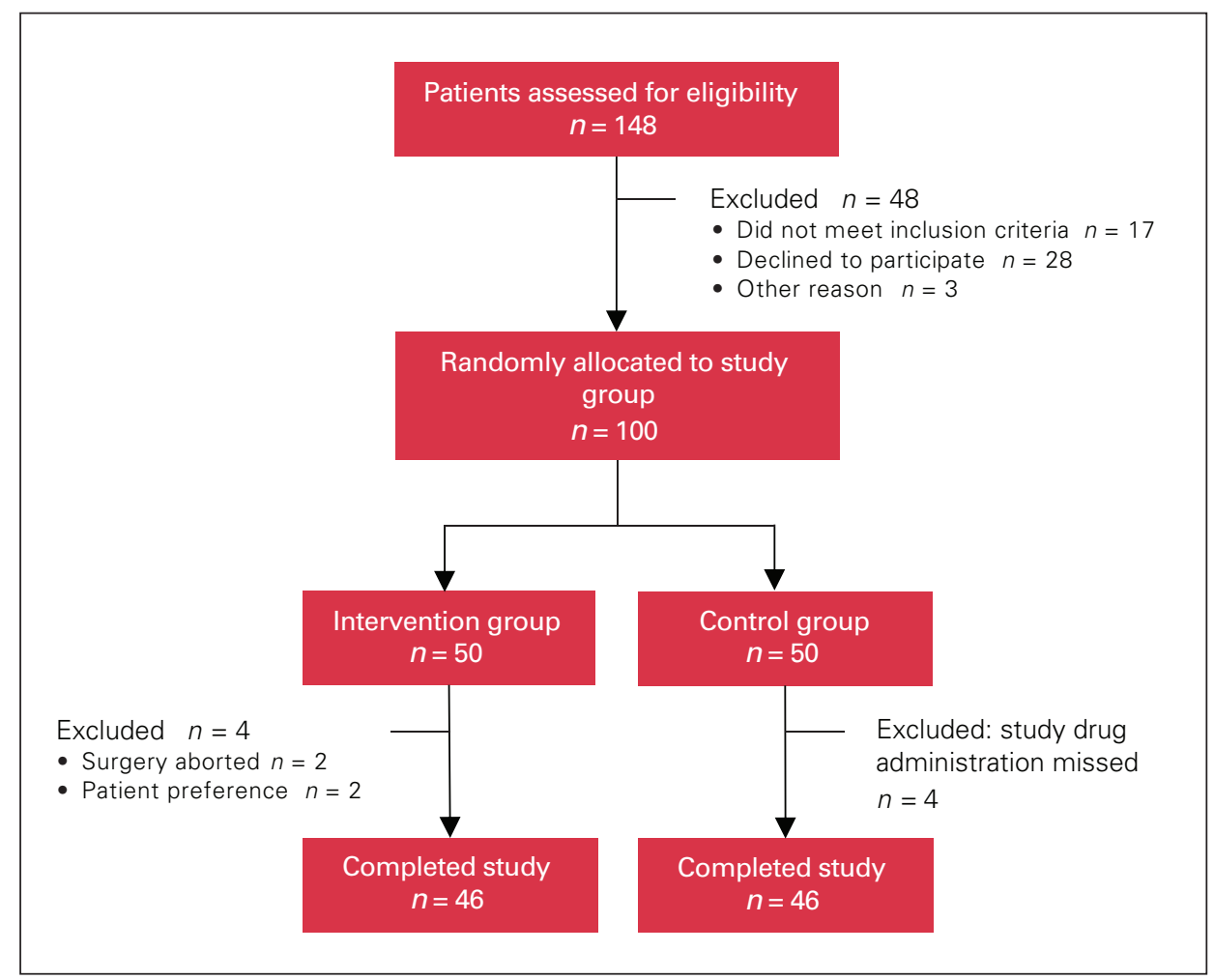

Fig. 1. Flow diagram showing patient allocation.

Table 1. Demographic characteristics, comorbidities, preoperative body mass index and intraoperative variables for patients undergoing bariatric surgery with or without intraperitoneal local anesthesia

\begin{tabular}{|c|c|c|c|}
\hline \multirow[b]{2}{*}{ Variable } & \multicolumn{2}{|c|}{ Group; no. (\%) of patients* } & \multirow[b]{2}{*}{$p$ value } \\
\hline & $\begin{array}{l}\text { IPLA } \\
n=46\end{array}$ & $\begin{array}{l}\text { Control } \\
n=46\end{array}$ & \\
\hline \multicolumn{4}{|l|}{ Preoperative } \\
\hline Age, mean $\pm S D, y r$ & $44.4 \pm 9.42$ & $45.1 \pm 9.44$ & 0.8 \\
\hline Female gender & $38(83)$ & $39(85)$ & 0.8 \\
\hline Body mass index, mean $\pm \mathrm{SD}$ & $48.6 \pm 6.10$ & $45.8 \pm 6.07$ & 0.04 \\
\hline History of pain syndromes & 2 (4) & $9(20)$ & 0.02 \\
\hline History of asthma & $14(30)$ & $6(13)$ & 0.04 \\
\hline History of cardiovascular disease & $15(33)$ & $24(52)$ & 0.06 \\
\hline History of arthritis & $22(48)$ & $22(48)$ & 1.0 \\
\hline Receiving diabetic medications & $12(26)$ & $18(39)$ & 0.2 \\
\hline Receiving pain medications & $20(43)$ & $22(48)$ & 0.7 \\
\hline Cesarean section & $9(20)$ & $12(26)$ & 0.46 \\
\hline Laparoscopic cholecystectomy & $13(28)$ & $9(20)$ & 0.3 \\
\hline Any other prior operation & $44(96)$ & $44(96)$ & 1.0 \\
\hline \multicolumn{4}{|l|}{ Intraoperative } \\
\hline Length of operation, mean $\pm \mathrm{SD}, \mathrm{h}$ & $1.85 \pm 0.38$ & $1.86 \pm 0.40$ & 0.9 \\
\hline Liver laceration & $3(6)$ & $5(11)$ & 0.7 \\
\hline Cholecystectomy & $0(0)$ & $1(2)$ & 1.0 \\
\hline
\end{tabular}

Analgesic consumption was similar for the intervention and control groups (Table 3). There was wide variation in the mean dosages of the analgesics used, without any over- all statistically significant or clinically relevant differences.

There were no differences at baseline or on the first postoperative day between the 2 groups in PEF, 6MWT 


\begin{tabular}{|c|c|c|c|}
\hline \multirow{2}{*}{$\begin{array}{l}\text { Time since } \\
\text { surgery, h }\end{array}$} & \multicolumn{2}{|c|}{ Group; pain score, ${ }^{*}$ mean $\pm S D$} & \multirow[b]{2}{*}{$p$ value } \\
\hline & IPLA & Control & \\
\hline $1-8$ & $3.4948 \pm 1.4309$ & $3.5643 \pm 1.7125$ & 0.7 \\
\hline $9-24$ & $3.6746 \pm 1.769$ & $3.5118 \pm 1.898$ & 0.2 \\
\hline $54-48$ & $3.0489 \pm 1.7787$ & $2.9587 \pm 1.8288$ & 0.7 \\
\hline \multicolumn{4}{|c|}{$\begin{array}{l}\text { IPLA = intraperitoneal local anesthetic; } S D=\text { standard deviation. } \\
{ }^{*} \text { As assessed with a numeric pain scale ranging from } 0 \text { (no pain at all) to } 10 \text { (worst pain } \\
\text { imaginable). }\end{array}$} \\
\hline
\end{tabular}

\begin{tabular}{|c|c|c|c|}
\hline \multirow[b]{2}{*}{ Analgesic } & \multicolumn{2}{|c|}{ Group; amount, mean $\pm \mathrm{SD}$, mg } & \multirow[b]{2}{*}{$p$ value } \\
\hline & IPLA & Control & \\
\hline Acetaminophen & $690.948 \pm 781.883$ & $698.964 \pm 800.452$ & 0.9 \\
\hline Ketorolac & $4.1148 \pm 7.9937$ & $4.7555 \pm 9.0907$ & 0.4 \\
\hline Hydromorphone & $12.9804 \pm 19.2248$ & $11.5646 \pm 14.7476$ & 0.4 \\
\hline Tramadol & $49.9913 \pm 58.7218$ & $49.4422 \pm 60.8887$ & 0.9 \\
\hline
\end{tabular}

\begin{tabular}{|c|c|c|c|c|c|c|}
\hline \multirow[b]{2}{*}{ Time } & \multicolumn{2}{|c|}{$\mathrm{PEF}$, mean $\pm \mathrm{SD}, \mathrm{L} / \mathrm{min}$} & \multicolumn{2}{|c|}{$6 \mathrm{MWT}$ score, mean $\pm \mathrm{SD}, \mathrm{m}$} & \multicolumn{2}{|c|}{ QoR-40 score, ${ }^{*}$ mean $\pm S D$} \\
\hline & Control & IPLA & Control & IPLA & Control & IPLA \\
\hline Baseline & $365 \pm 101.2$ & $369.9 \pm 126.2$ & $382.2 \pm 67.2$ & $367.7 \pm 73.5$ & $186.5 \pm 11.8$ & $187.1 \pm 9.2$ \\
\hline \multicolumn{7}{|c|}{ Postoperatively } \\
\hline Day 1 & $240.56 \pm 93.6$ & $241.73 \pm 79.8$ & $208.8 \pm 94.3$ & $182.4 \pm 97.1$ & $163 \pm 16.5$ & $163.6 \pm 14.7$ \\
\hline Day 7 & - & - & $373.3 \pm 68.12$ & $352.4 \pm 90.2$ & $183.5 \pm 14.2$ & $182.7 \pm 14.1$ \\
\hline
\end{tabular}

score or QoR-40 score (Table 4). All patients, irrespective of their group allocation, showed a decrease in these outcomes in the first 24 hours postoperatively, and then 6MWT and QoR-40 scores had recovered by the seventh postoperative day. There were no differences between the groups, and all patients showed a return to their baseline functional status on the seventh postoperative day.

\section{Discussion}

In this pilot study evaluating the clinical efficacy of adding IPLA to a standardized ERABS protocol, we found no significant differences between the patients who received IPLA and those who did not in pain scores, analgesic consumption (even though there were more patients with a history of chronic pain in the control group than in the intervention group), PEF on postoperative day 1, and 6MWT and QoR-40 scores on postoperative day 7.

The negative results of this clinical trial must be interpreted in the context of low pain scores and analgesic consumption in both groups. All the study patients, irrespective of their group allocation, received a standardized opioid-sparing anesthetic and analgesic protocol. Multimodal analgesia itself reduces pain scores, opioid analgesic consumption and adverse effects after bariatric surgery. ${ }^{13}$ In addition, our standardized ERABS protocol included both ketamine and dexmedetomidine, which are individually known to reduce pain and minimize opioid analgesic consumption without increased sedation or respiratory depression. These are highly desirable features in patients with extreme obesity and especially after bariatric surgery. ${ }^{13,14}$ Beyond their excellent analgesic properties, these agents can contribute to ERABS outcomes through reduc- tion in opioid-related adverse effects, including sedation, respiratory depression, nausea and vomiting. ${ }^{13-15}$ Perioperative care that includes ERABS protocols and implements opioid-sparing (or opioid-free) anesthetic strategies is a major evolution in bariatric anesthesia. ${ }^{16}$ In this paradigm shift toward minimizing opioid use after bariatric surgery, the role of regional anesthesia techniques continues to need evaluation. Our study contributes to this area of research by confirming lack of benefit of IPLA in improving outcomes. Nonetheless, our study protocol and methodology will be useful to guide further research in perioperative pain management techniques in ERABS.

The results of this study also need to be considered in the larger context of being conducted in a university level tertiary referral hospital that is a provincially designated Bariatric Centre of Excellence. Our well-established bariatric surgical program has provided elective bariatric surgery to more than 7000 patients since 2009. As observed elsewhere with well-established ERAS centres, beyond a certain point, further reductions in traditional measures (e.g., length of stay, complications and readmission rates) may not be possible. ${ }^{17}$ Nevertheless, over time and through improvements in the protocols, our research and that of other investigators ${ }^{18}$ draw attention to the role of functional prehabilitation by working toward improving patient education, engagement and empowerment.

Our ERABS patient education includes both hard-copy booklets and online resources that are combined with personalized teaching through in-person sessions. All these provide the patient with the information required to participate in an ERABS program. Furthermore, using tools such as the 6MWT and PEF further engages patients and allows them to participate actively in their own care. We 
chose these 2 measures as, in our opinion, they best reflect the ERABS outcomes we would like all our patients to achieve before and after bariatric surgery, including deep breathing and adequate ambulation. Since patients are not blinded to these measures of their level of activity before surgery, they are motivated to achieve them after surgery. Finally, it is our impression that these simple functional outcome measures empower patients to achieve the central goal of ERABS, which is a return to baseline.

Although further research and implementation are required to standardize these and other ERABS tools, this study is an important first step in that direction. Overall, ERABS should improve patient safety and outcomes after bariatric surgery and will benefit patients, providers and health care systems.

\section{Limitations}

The study was initially designed as a pilot study, which is a limitation. However, we were able to recruit the appropriate number of patients and executed the of methodology successfully, and the results are strongly suggestive of lack of benefit.

\section{Conclusion}

In the setting of a standardized ERABS protocol, this prospective double-blind randomized pilot trial showed no additional clinical benefit of IPLA in patients undergoing bariatric surgery. This shows the need for alternative methods for pain control after bariatric surgery.

Affiliations: From the Department of Surgery, The Ottawa Hospital, Ottawa, Ont. (Jarrar, Wu, Neville, Yelle, Mamazza); and the Department of Anesthesiology, The Ottawa Hospital, Ottawa, Ont. (Eipe).

Competing interests: None declared.

Contributors: All authors designed the study. A. Jarrar, R. Wu, A. Neville, J.-D. Yelle and J. Mamazza acquired the data, which A. Jarrar, N. Eipe, R. Wu and J. Mamazza analyzed. A. Jarrar and N. Eipe wrote the manuscript, which all authors critically revised. All authors gave final approval of the article to be published.

Content licence: This is an Open Access article distributed in accordance with the terms of the Creative Commons Attribution (CC BYNC-ND 4.0) licence, which permits use, distribution and reproduction in any medium, provided that the original publication is properly cited, the use is noncommercial (i.e., research or educational use), and no modifications or adaptations are made. See: https://creativecommons. org/licenses/by-nc-nd/4.0/.

Funding: This study was funded by the Department of Surgery, The Ottawa Hospital.

\section{References}

1. Ljungqvist O, Scott M, Fearon KC. Enhanced Recovery after Surgery: a review. FAMA Surg 2017;152:292-8.
2. Mannaerts GH, van Mil SR, Stepaniak PS, et al. Results of implementing an Enhanced recovery after bariatric surgery (ERABS) protocol. Obes Surg 2016;26:303-12.

3. Budiansky AS, Margarson MP, Eipe N. Acute pain management in morbid obesity - an evidence based clinical update. Surg Obes Relat Dis 2017;13:523-32.

4. Awad S, Carter S, Purkayastha S, et al. Enhanced recovery after bariatric surgery (ERABS): clinical outcomes from a tertiary referral bariatric centre. Obes Surg 2014;24:753-8.

5. Kahokehr A, Sammour T, Srinivasa S, et al. Systematic review and meta-analysis of intraperitoneal local anaesthetic for pain reduction after laparoscopic gastric procedures. Br F Surg 2011;98:29-36.

6. Boddy AP, Mehta S, Rhodes M. The effect of intraperitoneal local anesthesia in laparoscopic cholecystectomy: a systematic review and meta-analysis. Anesth Analg 2006;103:682-8.

7. Symons JL, Kemmeter PR, Davis AT, et al. A double-blinded, prospective randomized controlled trial of intraperitoneal bupivacaine in laparoscopic Roux-en-Y gastric bypass. 7 Am Coll Surg 2007;204: 392-8.

8. Alkhamesi NA, Kane JM, Guske PJ, et al. Intraperitoneal aerosolization of bupivacaine is a safe and effective method in controlling postoperative pain in laparoscopic Roux-en-Y gastric bypass. 7 Pain Res 2008;1:9-13.

9. Wu R, Haggar F, Porte N, et al. Assessing the feasibility of a randomised, double-blinded, placebo-controlled trial to investigate the role of intraperitoneal ropivacaine in gastric bypass surgery: a protocol. BMF Open 2014;4:e005823.

10. Kucuk C, Kadiogullari N, Canoler O, et al. A placebo-controlled comparison of bupivacaine and ropivacaine instillation for preventing postoperative pain after laparoscopic cholecystectomy. Surg Today 2007;37:396-400.

11. Knudsen K, Beckman Suurküla M, Blomberg S, et al. Central nervous and cardiovascular effects of i.v. infusions of ropivacaine, bupivacaine and placebo in volunteers. Br F Anaesth 1997;78:507-14.

12. Gornall BF, Myles PS, Smith CL, et al. Measurement of quality of recovery using the QoR-40: a quantitative systematic. Br 7 Anaesth 2013;111:161-9.

13. Feld JM, Hoffman WE, Stechert MM, et al. Fentanyl or dexmedetomidine combined with desflurane for bariatric surgery. 7 Clin Anesth 2006;18:24-8.

14. Ziemann-Gimmel P, Hensel P, Koppman J, et al. Multimodal analgesia reduces narcotic requirements and antiemetic rescue medication in laparoscopic Roux-en-Y gastric bypass surgery. Surg Obes Relat Dis 2013;9:975-80.

15. Ziemann-Gimmel P, Goldfarb AA, Koppman J, et al. Opioid-free total intravenous anaesthesia reduces postoperative nausea and vomiting in bariatric surgery beyond triple prophylaxis. $\mathrm{Br} 7$ Anaesth 2014;112:906-11.

16. Mulier J. Opioid free general anesthesia: A paradigm shift? Rev Esp Anestesiol Reanim 2017;64:427-30.

17. Abeles A, Kwasnicki RM, Darzi A. Enhanced recovery after surgery: current research insights and future direction. World 7 Gastrointest Surg 2017;9:37-45.

18. Minnella EM, Bousquet-Dion G, Awasthi R, et al. Multimodal prehabilitation improves functional capacity before and after colorectal surgery for cancer: a five-year research experience. Acta Oncol 2017; 56:295-300. 